Reprod. Nutr. Dévelop., 1988, 28 (6 B), 1741-1746

\title{
La décroissance du taux d'HCG après traitement cœlioscopique conservateur de la grossesse extra-utérine
}

\author{
J. L. POULY, G. MAGE, H. MANHES, Françoise GACHON (*), Ginette GAIL- \\ LARD $\left(^{*}\right)$, M. A. BRUHAT \\ Service de Gynécologie-Obstétrique, CHU, Polyclinique B.P. 69, \\ 5, boulevard Charles-de-Gaulle \\ 63033. Clermont-Ferrand Cedex, France. \\ ( ${ }^{*}$ Laboratoires /sotopes in vitro, Centre Jean Perrin. \\ 30, place Henri-Dunant. \\ 63011. Clermont-Ferrand Cedex
}

Summary. HCG decrease after laparoscopic treatment of ectopic pregnancy.

HCG levels have been studied in 76 patients following laparoscopic treatment of ectopic pregnancy. The decrease of HCG was the same as after conservative treatment using laparotomy. This study shows that early detection of treatment failure is possible from the third or fifth post-operative day onwards. The authors report a scheme for the post-operative follow-up after laparoscopic treatment of ectopic pregnancy based on the rate of HCG decrease.

\section{Introduction.}

Nous avons rapporté précédemment notre expérience du traitement cœlioscopique de la grossesse extra-utérine (Bruhat et al., Manhes et al., 1982). Cette technique paraît promise à un grand avenir car elle évite une laparotomie et donc diminue le désagrément pour la patiente et le coût pour la société. Par ailleurs ce traitement préserve bien la fertilité ultérieure des patientes (Mage et al., 1983; Decherney et al., 1981).

Le reproche principal fait à cette technique, est le risque d'échecs par rétention de trophoblaste dans la trompe. Les premiers échecs sont survenus à une époque où le dosage de l'HCG n'était pas encore un examen courant, seuls les signes cliniques après le $20^{\mathrm{e}}$ jour postopératoire laissaient soupçonner cet échec. Depuis nous avons entrepris la surveillance postopératoire des patientes par le dosage de cette hormone.

\section{Matériel et méthodes.}

76 courbes de décroissance ont été étudiées: 69 correspondent à des cas traités avec succès dans le service de Gynécologie du CHU de Clermont-Ferrand entre janvier 1982 et juin 1985 (groupe A), et 7 aux cas de traitements avec 
échecs pour lequel nous disposons d'une courbe d'évolution du taux d'HCG (après 1979) (groupe B).

L'H.C.G. a été dosée par méthode radioimmunologique à partir de la trousse «H.C.G.-R.I.A.» distribuée par le C.I.S. France. Les valeurs normales du laboratoire, en dehors de la grossesse, sont inférieures à $1 \mathrm{ng} / \mathrm{ml}$ (référence: $2^{e}$ standard international MRC 75/537). $1 \mathrm{ng}$ correspond à $8,6 \mathrm{mUl}$ du MRC 75/537).

Pour chacune de ces patientes nous disposons d'un dosage de départ à partir d'un échantillon prélevé dans les $24 \mathrm{~h}$ précédant le traitement. Les échantillons ultérieurs ont été prélevés à des jours variables selon les patientes du $2^{\mathrm{e}}$ au $30^{\mathrm{e}}$ jour postopératoire jusqu'à négativation du taux $(<1 \mathrm{ng} / \mathrm{ml})$. Par approximation ils ont été interprétés comme réalisés aux jours : 2, 3, 4, 5, 6, 7, 10, 12, 14, 16, 20, 25, 30. Par ailleurs lorsqu'un taux est devenu négatif à un jour postopératoire donné, les taux des jours suivants sont considérés comme négatifs même si les dosages n'ont pas été réalisés.

\section{Résultats.}

Dans le groupe A (cas de succès opératoires) les taux de départ sont extrêmement dispersés : la moyenne est de $871 \mathrm{ng} / \mathrm{ml}$, l'écart-type est de 1771 . Les valeurs extrêmes retrouvées sont de 3,4 et $12470 \mathrm{ng} / \mathrm{ml}$. La figure 1 et le tableau 1 montrent la décroissance rapide du taux d'HCG, mais les valeurs absolues étant très dispersées, l'étude a été reprise en considérant chaque valeur en pourcentage de la valeur initiale. Ces résultats sont rapportés sur le tableau 1.

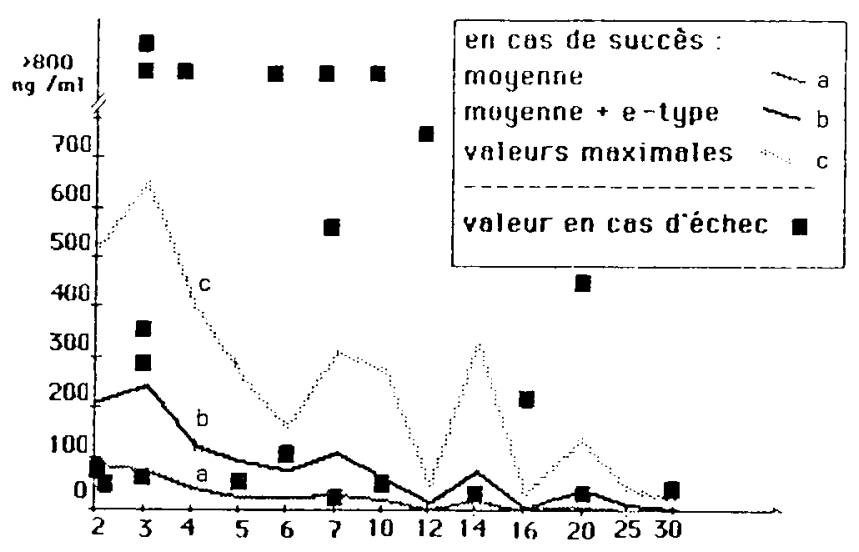

FIG. 1. - Décroissance, en fonction du jour postopératoire, du taux d'HCG, en valeur absolue, dans les cas de succès (ligne de moyenne, moyenne + écart-type, plus grandes valeurs observées) et dans le cas d'échecs (carré noir).

Cet artifice de calcul montre que la disparité des valeurs observées tient surtout à la différence des taux de départ. 
TABLEAU 1

Valeurs absolues et relatives du taux d'HCG en fonction du jour postopératoire : (moyenne, écart-type et plus grande valeur rencontrée).

\begin{tabular}{|c|c|c|c|c|c|c|c|}
\hline \multirow[t]{2}{*}{ Jour } & \multirow[t]{2}{*}{$N$} & \multicolumn{2}{|c|}{ Valeurs absolues } & \multirow[b]{2}{*}{ Max. } & \multicolumn{2}{|c|}{ Valeurs relatives } & \multirow[b]{2}{*}{ Max } \\
\hline & & $\mathrm{m}$. & e.t. & & $\mathrm{m}$. & e.t. & \\
\hline 2 & 32 & 90,2 & 125,6 & 516 & 17,4 & 12,6 & 60,6 \\
\hline 3 & 29 & 79,9 & 165,1 & 652 & 8,5 & 6,5 & 21,2 \\
\hline 4 & 20 & 42,9 & 87,1 & 398 & 4,6 & 6,2 & 27,9 \\
\hline 5 & 12 & 27,1 & 71,9 & 264 & 2,7 & 4,3 & 13,2 \\
\hline 6 & 13 & 26,1 & 52,4 & 164 & 1,9 & 2,7 & 13,1 \\
\hline 7 & 23 & 30,5 & 86,1 & 311 & 1,9 & 3,1 & 8 \\
\hline 10 & 32 & 18,7 & 50,9 & 277 & 1,2 & 2,1 & 10,8 \\
\hline 12 & 23 & 4,2 & 11,5 & 49 & 0,8 & 2,7 & 13,3 \\
\hline 14 & 29 & 18,8 & 61,3 & 329 & 0,6 & 1,7 & 8,7 \\
\hline 16 & 29 & 1,9 & 6,2 & 30 & 0,2 & 0,6 & 2,9 \\
\hline 20 & 37 & 11 & 31,8 & 141 & 0,3 & 1 & 4,8 \\
\hline 25 & 34 & 2,3 & 9,1 & 43,5 & 0 & 0,2 & 0,9 \\
\hline 30 & 37 & 1 & 3,1 & 21 & 0 & 0,2 & 1 \\
\hline
\end{tabular}

La négativation du taux d'HCG a été admise pour des taux très bas $(<1 \mathrm{ng} / \mathrm{ml})$. Elle n'a pu être appréciée que chez 37 patientes. La négativation est obtenue dans $89,2 \%$ des cas au $30^{\mathrm{e}}$ jour, la valeur maximale observée ce jour-là étant de $21 \mathrm{ng} / \mathrm{ml}$ pour un taux de départ à $12470 \mathrm{ng} / \mathrm{ml}$. La négativation la plus précoce observée se situe au $2^{\mathrm{e}}$ jour pour un taux de départ de $3,4 \mathrm{ng} / \mathrm{ml}$. La médiane de négativation se situe au jour 12,34.

Afin de s'assurer que le taux de départ n'influe pas sur la décroissance exprimée en pourcentage, cette étude a été menée en considérant les cas d'une part des 36 valeurs de départ les plus élevées, d'autre part des 33 valeurs de départ les plus basses. La frontière entre ces 2 groupes se situe à $200 \mathrm{ng} / \mathrm{ml}$. Les résultats sont rapportés dans le tableau 2. Statistiquement il n'existe aucune différence significative dans les décroissances relatives observées (test de Student-Fischer). La seule différence réside dans le taux de négativation. La négativation est obtenue dans $100 \%$ des cas $(20 / 20)$ au $20^{\mathrm{e}}$ jour dans le groupe à taux de départ bas avec une médiane à 6,75 jours alors que dans le groupe à taux de départ élevé, le taux de négativation est de $76,5 \%(13 / 17)$ au $30^{\mathrm{e}}$ jour, la médiane se situant à 17 jours. La différence est significative aux jours $3,4,5,6,7,10,12,14,16,20$, 25 (Test du H khi 2).

L'intégration des données de décroissance relative permet de déduire la courbe de régression de la moyenne et de l'écart-type qui répondent à une équation du type: $Y=A X+B$

avec $Y=$ taux relatif d'HCG, $X=1$ /jour postopératoire, $A=27,74$, $B=-1,022$.

L'écart-type de la décroissance relative répond à la formule : Ecart-type de la décroissance relative $=24 /$ (jour) . 
TABLEAU 2

Comparaison de la décroissance relative entre le groupe à taux d'HCG de départ bas $(<199 \mathrm{ng} / \mathrm{ml})$ et celui à taux de départ élevé $(<199 \mathrm{ng} / \mathrm{ml})$.

\begin{tabular}{|c|c|c|c|c|c|c|c|}
\hline \multirow{3}{*}{$\begin{array}{c}\text { Jour } \\
\text { taux de } \\
\text { base }\end{array}$} & \multicolumn{3}{|c|}{ Groupe à taux bas } & \multirow[b]{2}{*}{ nbre } & \multicolumn{2}{|c|}{ Groupe à taux éleve } & \multirow{3}{*}{$\begin{array}{c}p \\
t \text { test } \\
<0,0001\end{array}$} \\
\hline & nbre & $\mathrm{m}$. & e.t. & & $\mathrm{m}$. & e.t. & \\
\hline & 33 & 80,8 & 68,4 & 36 & 1494 & 2183 & \\
\hline 2 & 15 & 17 & 16,1 & 17 & 17,7 & 8,2 & NS \\
\hline 3 & 18 & 7,7 & 7,3 & 11 & 9,8 & 4,6 & NS \\
\hline 4 & 10 & 1,4 & 2,8 & 10 & 7,9 & 7 & NS \\
\hline 5 & 8 & 0 & 0 & 4 & 8,1 & 3,4 & NS \\
\hline 6 & 10 & 1 & 2,1 & 3 & 4,9 & 2,3 & NS \\
\hline 7 & 16 & 1,3 & 3,2 & 7 & 3,3 & 2,5 & NS \\
\hline 10 & 19 & 0,3 & 0,8 & 13 & 2,5 & 2,7 & NS \\
\hline 12 & 17 & 0 & 0 & 6 & 2,9 & 4,7 & NS \\
\hline 14 & 17 & 0 & 0 & 6 & 1,4 & 2,3 & NS \\
\hline 16 & 18 & 0 & 0 & 11 & 0,5 & 0,9 & NS \\
\hline 20 & 20 & 0 & 0 & 17 & 0,8 & 1,4 & NS \\
\hline 25 & 20 & 0 & 0 & 14 & 0.1 & 0.2 & NS \\
\hline 30 & 20 & 0 & 0 & 17 & 0,1 & 0,2 & NS \\
\hline
\end{tabular}

Dans le groupe des échecs du traitement (groupe B), 22 valeurs sont connues pour 7 patientes. Le taux moyen de départ est plus élevé que dans le groupe $A$ : $1152,3 \mathrm{ng} / \mathrm{ml}$ (écart-type $=1715$ ) ( $p<0,0001$ ), les valeurs extrêmes observées étant 5267 et $75 \mathrm{ng} / \mathrm{ml}$. L'analyse statistique des valeurs absolues amène peu de renseignement du fait de la grande dispersion de celles-ci. Néanmoins le report des chiffres sur la courbe de décroissance en valeurs absolues (fig. 1) montre que 4 données (18,2\%) sont inférieures à la moyenne générale, $3(13,6 \%)$ sont comprises entre la moyenne et la moyenne + écart-type et $3(13,6 \%)$ sont supérieures à la moyenne + écart-type mais inférieures aux chiffres maximum rencontrés dans le groupe $A$.

Par contre, l'analyse de la décroissance relative est beaucoup plus intéressante. Les extrêmes observées sont de $15,7 \%$ au jour 7 et $428,8 \%$ au jour 20 . Au jour 3, 5 valeurs ont été observées avec une valeur moyenne de 100,2 (écarttype $=85,6)$. La différence est très significative par rapport au groupe $A$ $(p<0,000001)$.

Par ailleurs le report des valeurs de décroissance relative sur la courbe du groupe A montre qu'elles sont toutes supérieures à la moyenne + écart-type et que seules $3(13,6 \%)$ sont comprises entre la moyenne + écart-type et les chiffres maximum rencontrés dans le groupe $A$. Toutes ces patientes ont eu un dosage au jour 2 ou au jour 3 et dans tous les cas la décroissance relative était supérieure à la moyenne + écart-type du groupe $A$.

En dernier lieu, le report de ces valeurs dans la courbe de régression (fig. 2) montre que toutes étaient supérieures à la moyenne +2 écarts-types sauf une égale à la moyenne + écart-type au jour 3. Dans ce dernier cas, la surveillance aux jours 5 et 7 a donné des résultats supérieurs à la moyenne +2 écarts-types. 


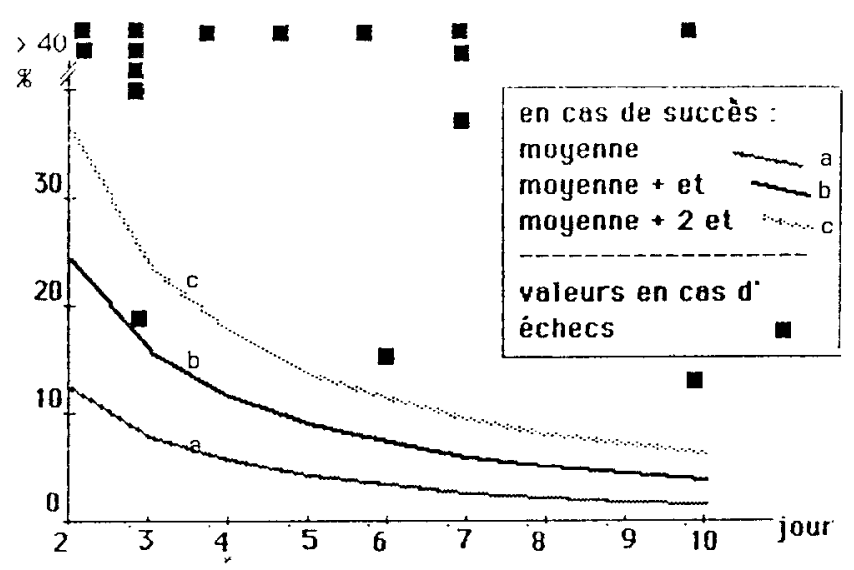

FIG. 2. - Report des valeurs relatives d'HCG, en fonction du jour postopératoire, dans les cas d'échecs (carré noir) sur la courbe de régression des valeurs relatives en cas de succès (moyenne, moyenne + écart-type, moyenne + 2 écarts-types).

\section{Discussion.}

La décroissance du taux d'HCG est indépendante du taux de départ. La seule différence qui existe se situe dans le délai de négativation et cette différence statistique est artificiellement majorée par la méthode de calcul qui admet la négativation pour un taux non strictement nul.

La décroissance semble se faire selon une double courbe: dans les 48 premières heures la demi-vie est d'environ 19,4 h, au-delà la demi-vie est de $63,9 \mathrm{~h}$.

Ces résultats sont légèrement différents de ceux rapportés par Steier et al., (1984) dans 35 cas de grossesses extra-utérines traitées conservativement par laparotomie. Celui-ci retrouve une médiane de négativation à 8,5 jours avec des extrêmes de 1 à 31 jours. La décroissance rapportée est par ailleurs superposable. La différence entre nos chiffres et ceux de Steier s'explique par 2 éléments : le taux de négativation que nous avons considéré est inférieur au sien $(8,6 \mathrm{UI} / \mathrm{I}$ contre $10 \mathrm{UI} / \mathrm{I}$ ) et surtout la moyenne des taux de base est plus forte dans notre groupe témoin $(871 \mathrm{ng} / \mathrm{ml}$ soit $7490 \mathrm{UI} / \mathrm{I}$ contre $1288 \mathrm{UI} / \mathrm{I}$ pour Steier).

Les résultats confirment que l'échec du traitement cœelioscopique de grossesse extra-utérine est dû à une ablation incomplète du trophoblaste et non à une hémorragie secondaire bien que celle-ci en soit la traduction clinique tardive.

La surveillance postopératoire de la décroissance du taux d'HCG peut être allégée en fonction des données de la figure 3. La détection précoce des échecs est possible au plus tard au jour 4.

Un dosage d'HCG est effectué au $2^{\mathrm{e}}$ ou $3^{\mathrm{e}}$ jour postopératoire ; ce chiffre est divisé par le taux de départ et multiplié par 100. Le chiffre obtenu est reporté sur la courbe où 4 zones sont définies : la zone $A$ correspond au cas le plus fréquent, le traitement est un succès, aucun contrôle ultérieur n'est nécessaire. La zone $B$ : le traitement est très vraisemblablement un succès néanmoins un contrôle entre 


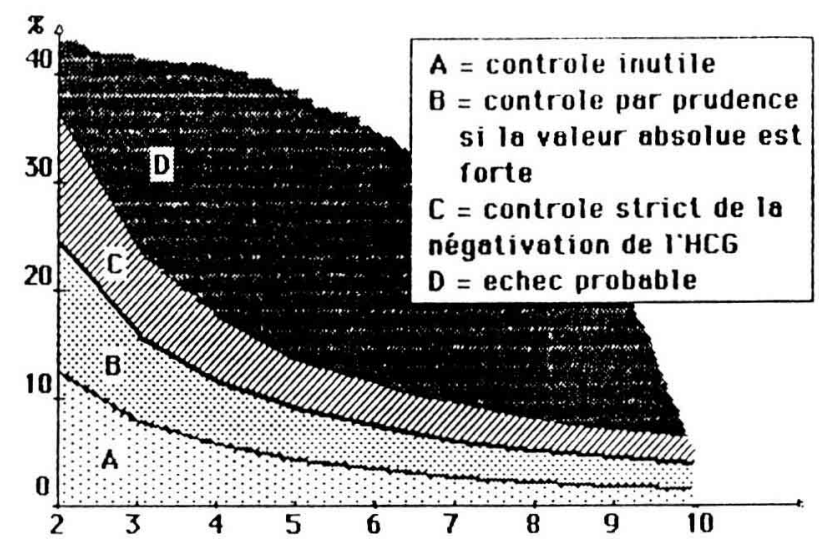

FIG. 3. - Schématisation de la conduite à tenir en fonction de la décroissance relative du taux d'HCG, dans les suites du traitement cœelioscopique de la grossesse extrautérine.

le $7^{\mathrm{e}}$ et le $10^{\mathrm{e}}$ jour postopératoire est préférable. La zone $\mathrm{C}$ : il existe un doute quant à l'efficacité du traitement. La probabilité d'échec est relativement élevée. La décroissance doit être contrôlée de façon stricte tous les 2 jours jusqu'à ce que l'évolution se fasse nettement dans un sens ou l'autre. La zone D: l'échec du traitement est probable, il faut le pallier rapidement sans attendre les conséquences aussi bien générales que locales. La thérapeutique de "rattrapage » peut se concevoir différemment: reprise immédiate par cœlioscopie ou laparotomie, prescription de RU 486, prescription de Methotrexate...

$27^{e}$ Réunion de la Société française pour l'Etude de la Fertilité. Paris, 29, 30 sept., $1^{\text {er }}$ oct. 1988.

\section{Références}

BRUHAT M. A., MANHES H., MAGE G., POULY J. L., 1980. Treatment of ectopic pregnancy by means of laparoscopy. Fertil. Steril., 33, 411-414.

DECHERNEY A. H., ROMERO R., NAFTOLIN F., 1981. Surgical management of unruptured ectopic pregnancy. Fertil. Steril. 35, 26-24.

MAGE G., MANHES H., POULY J. L., ROPERT J. F., BRUHAT M. A., 1983. J. Gyn. Obst. Biol. Reprod. 12, 775-778.

MANHES H., MAGE G., POULY J. L., ROBERT J. F., BRUHAT M. A., 1983. Traitement cœlioscopique de la grossesse extrautérine non rompue : améliorations techniques. Nouv. Presse méd. 12, 1431-1432.

STEIER J. A., BERGSJO P., MYKING O. L., 1984. Human gonadotrophin in maternal plasma after induced abortion, spontaneous abortion and removed ectopic pregnancy. Obstet. Gynecol., 64, 391-394. 